\title{
Prótesis 3D para corregir eje corporal y equilibro en lechuza de campanario y cernícalo americano
}

\author{
Estay, S.A., ${ }^{1,2}$ Oidor, M.A. ${ }^{1}$ Ramírez, J.S. ${ }^{2}$ \\ ${ }^{1}$ Benemérita Universidad Autónoma de Puebla, México. \\ ${ }^{1,2}$ Centro de Conservación de Vida Silvestre "Konkon", \\ Totimehuacan, Puebla. E-mail: andres.estay@acorreo.buap.mx
}

\begin{abstract}
Resumen
Estay, S.A.; Oidor, M.A.; Ramírez, J.S.: Prótesis 3D para corregir eje corporal y equilibrio en lechuza de campanario y cernicalo americano. Rev. Vet. 32: 1, 95-100, 2021. La amputación de miembros pélvicos en aves rapaces, debe ser evaluada con detenimiento y considerada como el último recurso para salvar la vida del ave, debido a que posterior a la amputación, pueden derivarse una serie de factores degenerativos, como la alteración de eje corporal, distribución inadecuada del peso corporal y pododermatitis. Una buena alternativa para contrarrestar estos problemas, es el uso de prótesis, que busquen recuperar el eje corporal y mantener una buena postura en el ave. El uso de impresoras 3D para este tipo de proyectos, ofrece una alternativa más accesible y sencilla debido a su versatilidad tanto en diseño como en materiales. El presente proyecto pone a prueba el diseño de una prótesis 3D y documenta la corrección de eje corporal e incorporación de la prótesis en las actividades diarias de un cernícalo americano (Falco sparverius) y una lechuza de campanario (Tyto $a l b a$ ), ambas con una amputación tibiotarsal en su tercio distal. A pesar de que la utilización de prótesis no permite la reintroducción de las aves al medio ambiente, ofrece una oportunidad para integrar dichas aves en programas de conservación, tales como reproducción en cautiverio o educación ambiental.
\end{abstract}

Palabras clave: impresión 3D, prótesis, raptor, cernícalo americano, lechuza de campanario.

\begin{abstract}
Estay, S.A.; Oidor, M.A.; Ramírez, J.S.: 3D protesis to correct body axis and balance in Barn owl and American kestrel. Rev. Vet. 32: 1, 95-100, 2021. The amputation of a pelvic limb in raptor, must be carefully evaluated and taken as the last possibility to save the life of the bird, because after the amputation, several degenerative factors can occur, such as alteration of the body axis, an incorrect distribution of weight and bumblefoot. A good alternative to counteract these problems is the use of prostheses that seek to help recover the body axis and maintain good posture. The use of 3D printers for this type of project offers a more accessible and easier to work alternative due to its versatility in both design and materials. The present project tests the design of a 3D prosthesis and documents the correction of the body axis and the modification of its use in the daily activities of an american kestrel and a barn owl with a tibiotarsal amputation in its distal third. Although the use of prostheses does not allow the reintroduction of the birds, it gives the opportunity to integrate them into conservation programs such as captivity reproduction or environmental education.
\end{abstract}

Key words: 3D printing, protesis, raptor, american kestrel, barn owl bumblefoot.

\section{INTRODUCCIÓN}

Las aves rapaces, tanto diurnas como nocturnas, en vida libre pueden presentar una gran variedad de lesiones al momento de ser incorporadas a proyectos de rehabilitación: electrocución, lesiones de armas de fuego, traumatismos de diferentes niveles, fracturas y astillamiento de huesos, entre otras ${ }^{1}$.

Cuando son aves en cautiverio, generalmente sufren lesiones relacionadas con un mal mantenimiento y una alimentación inadecuada que deriva en desnutrición generalizada, crecimiento anormal de pico y garras, lesiones derivadas de condiciones de transporte deficientes, heridas provocadas por mordeduras de otros animales en cautiverio o mascotas y fracturas provocadas por un mal manejo ${ }^{2,3}$.

Existen registros de aves en vida libre con lesiones en ojos ${ }^{4}$, pico ${ }^{5}$ y extremidades inferiores ${ }^{6,7,8}$, que demuestran que algunos individuos pueden compensar la pérdida de funcionalidad debido a una lesión y sobreviven, aunque se estima que por poco tiempo ${ }^{4}$. 


\section{Proyectos de manejo y conservación de recursos naturales}

Los estudios sobre diversidad han revelado que las actividades antropogénicas influyen de manera directa en la disminución del número de especies, en el tamaño y la variabilidad genética de las poblaciones silvestres y al mismo tiempo, provocan en otras especies incrementos de población y cambios en su distribución, en algunos casos de forma explosiva. Esta situación se manifiesta a nivel mundial y ha sido denominada como crisis de la biodiversidad ${ }^{9}$.

La sobreexplotación de los recursos naturales, el tráfico de especies, la destrucción del hábitat, el crecimiento de asentamientos urbanos, la introducción de especies exóticas al ecosistema, la caza furtiva y la contaminación ambiental, son algunas de las causas del declive de las poblaciones de animales de vida silvestre a nivel mundial ${ }^{10}$.

Dentro de las alternativas para contrarrestar los efectos de las actividades antropogénicas se plantea la necesidad de estrategias de conservación de recursos naturales in situ y ex situ, estableciendo proyectos que trabajen por la rehabilitación y liberación de ejemplares de vida silvestre lesionados, así como la reproducción en cautiverio para su posterior liberación ${ }^{11}$.

\section{Amputación}

Una gran cantidad de lesiones y factores pueden llevar a la amputación de un miembro pélvico en aves: fracturas compuestas, osteomielitis, infecciones medulares en fracturas expuestas, necrosis isquémica, gangrena, quemaduras, congelamiento, pododermatitis de grado $\mathrm{V}$ o mayor y corte de circulación por amarres inadecuados en cautiverio, son algunas de las más comunes ${ }^{12,3,13}$.

La amputación de un miembro pélvico debe ser evaluada con detenimiento y tomada como la última posibilidad para salvar la vida del ejemplar, debido a que posterior a la amputación, se pueden presentar varios factores degenerativos ${ }^{13,14,15}$.

La posibilidad de un ejemplar de sobrevivir y adaptarse a la amputación de una extremidad pélvica depende principalmente del tamaño del ave. Con posterioridad a la amputación, se debe poner especial atención en el desarrollo de pododermatitis, y en el cambio en el eje corporal (compensatorio a la restauración del equilibrio por parte del ejemplar), así como problemas de artritis en las articulaciones de la extremidad opuesta ${ }^{3}$.

De presentarse de forma continua el desarrollo de pododermatitis, debe considerarse la eutanasia del ejemplar ${ }^{13,16}$.

\section{Prótesis}

Las amputaciones de miembros de aves rapaces y el uso de prótesis, es un método para lograr la supervivencia de animales, que a pesar de no poder regresar a la vida silvestre, resulta valioso para reproducción en cautiverio, así como para animales con valor sentimen- tal o que puedan ser incorporados en proyectos de educación ambiental para la conservación ${ }^{17,18}$.

Una prótesis, es un artefacto diseñado para reemplazar alguna parte del cuerpo. En humanos la amputación de una extremidad y la fijación de prótesis han sido ampliamente estudiadas y pueden ser de dos tipos, endoesqueléticas y exoesqueléticas ${ }^{18,19,20}$

Las prótesis endoesqueléticas, implican la implantación a través de la piel y el hueso de un sistema de fijación para la prótesis. Las prótesis exoesqueléticas, también llamadas prótesis de socket, se pueden remover, no requieren de intervenciones quirúrgicas para utilizarse y constan de 3 partes principales, el socket o anclaje, la pierna y el pie, y en cada sección se deben incorporar uniones articuladas para facilitar el movimiento ${ }^{19}$.

\section{Casos clínicos. cernícalo americano (Falco sparverius)}

El cernícalo americano es el halcón más distribuido del continente americano, pero las poblaciones han disminuido en un $66 \%$ entre 1966 y 2014 . Se sabe que el $89.5 \%$ de los cernícalos americanos pertenecientes a Oregón, el este de Washington y Idaho son migratorios, por lo que migran principalmente al oeste de México para el invierno ${ }^{21}$.

Se han hecho estudios para tratar de determinar la causa en el descenso de esta especie, algunas causas potenciales incluyen la difusión del Virus del Oeste del Nilo (VON), aumentos en las poblaciones de halcón de Cooper (Accipitercooperii) y la perdida de ambientes propicios para la especie ${ }^{22}$.

Las poblaciones de cernícalo que anidaban en cajas nido comenzaron a experimentar disminuciones antes de la presencia del VON en el año de $1999^{23}$. En México, el Cernícalo americano es una de las especies más comunes en el comercio ilegal, debido a la abundancia de individuos que migran en época invernal, lo cual impacta negativamente en la población de dicha especie.

\section{Estudio del cernícalo americano}

En las instalaciones del Centro de Conservación de Vida Silvestre UMA "Konkon", se recibió un ejemplar de cernícalo americano hembra (Falco sparverius) que presentaba necrosis y gangrena en la extremidad inferior izquierda, en su tercio distal, a nivel de la articulación metatarso-falangiana (Figura 1).

El ave había sido mantenida en cautiverio, sin embargo no se aportó mayor información sobre la causa de la lesión. En una revisión inicial, el ave presentaba bajo peso y plumaje dañado, con exceso de grasa. También presentó pododermatitis grado 1 derivada de la incapacidad generada por la extremidad necrosada.

Se procedió a amputar la extremidad afectada en colaboración con el Hospital Veterinario de la Universidad de Puebla. El ejemplar fue sedado con Ketamina $10 \mathrm{mg} / \mathrm{kg}$ y Midazolam $0,25 \mathrm{mg} / \mathrm{kg}$ (aplicación intramuscular). 
Se mantuvo anestesiado con isoflurano con sonda endotraqueal CAM 1,44\%, $02200 \mathrm{ml} / \mathrm{kg} / \mathrm{min}$, VPP 8 $\mathrm{rpm} / 8-10 \mathrm{~cm}$ de agua. Se mantuvo una terapia de líquidos $20 \mathrm{ml} / \mathrm{kg} / \mathrm{hr}$ y solución de $\mathrm{NaCl}(0.9 \%)$. Las respuestas cardiaca, respiratoria y temperatura corporal fueron monitoreadas durante el procedimiento quirúrgico.

Las constantes fisiológicas se mantuvieron en rango y el ejemplar permaneció estable durante el procedimiento, la amputación se realizó desarticulando la articulación metatarso-falangiana. Una vez finalizada la amputación, la incisión fue cerrada con monofilamento absorbible y después de la cirugía el ejemplar fue medicado con Meloxicam $(0,5 \mathrm{mg} / \mathrm{kg})$ y Tramadol $(5 \mathrm{mg} /$ $\mathrm{kg}$ ) en dosis orales.

Después de 3 semanas, el muñón sanó completamente de la lesión y se empezó a formar la callosidad. A la sexta semana posterior a la amputación, la callosidad se encontraba completamente formada en el muñón y se procedió a iniciar la toma de datos y diseño de prótesis.

\section{Diagnóstico de eje corporal}

Una vez formada la callosidad en el muñón de la extremidad amputada, se evaluaron las alteraciones en el eje corporal del individuo, derivadas de la compensación de equilibrio ante la amputación de la extremidad.

Previo a la prueba de prótesis el ejemplar presentó una inclinación compensatoria de $6,7^{\circ}$ en dirección contraria a la extremidad amputada en el plano sagital, así como una inclinación compensatoria de $21,5^{\circ}$ en la extremidad sana, medida del centro de la base de la pata a la articulación pélvico-femoral (Figura 1).

\section{Toma de medidas de muñón}

Se tomaron las medidas de la extremidad amputada, analizando la circunferencia del muñón, circunferencia en la extremidad anterior al muñón, distancia a la articulación más cercana, superficie disponible para fijación de la prótesis, altura faltante de la extremidad

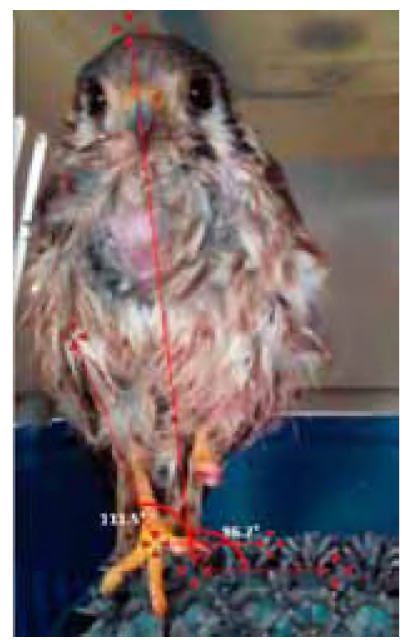

Figura 1. Ejemplar de Falco sparverius con amputación tarso-metatarsal, previo a la prueba de prótesis. Se muestran los ángulos de inclinación en el plano sagital y la extremidad derecha. y peso faltante de la extremidad. El muñón presentaba una forma irregular, debido a que se formó con una proyección de tejido (Tabla 1).

\section{Diseño de prótesis (Falco sparverius)}

Se diseñó la prótesis en 3D utilizando el programa AutoCAD $\mathbb{R}$. Se desarrolló un total de 3 diseños de socket y 1 diseño de poste/base para lograr una correcta fijación (Figura 2).

\section{Impresión de prótesis}

Se utilizó una impresora 3D marca Ultimaker 3 de doble extrusor, la articulación entre el poste y la base o pie, se incluyó dentro del mismo proceso de impresión, para evitar generar un punto débil al montar y desmontar la articulación. El peso de la prótesis se reguló disminuyendo el relleno de la impresión.

La impresión del poste y del socket se realizó con filamento de plástico rígido y filamento de plástico flexible respectivamente (Figura 2).

\section{Prueba de prótesis}

Durante la prueba el ejemplar comenzó a utilizar la prótesis gradualmente en sus actividades diarias, empezando por tomarla como apoyo al percharse y desplazarse, después comenzó a utilizarla para acomodar y sujetar su alimento y, gracias a la articulación, la utilizó también al impulsarse para saltar.

El plumaje del ejemplar mejoró considerablemente debido a un programa de alimentación, principalmente de ratones y pequeños roedores.

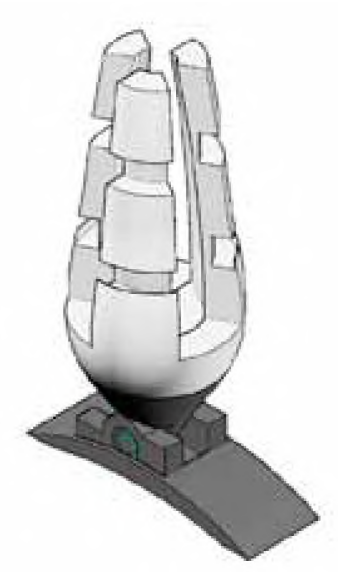

Figura 2. Modelo 3D de prótesis para cernícalo americano. El material blanco corresponde al plástico flexible y el gris al plástico rígido.

Tabla 1. Medidas de superficie de muñón y superficie y peso perdido (Falco sparverius)

\begin{tabular}{lc}
\hline circunferencia del muñón & $4,0 \mathrm{~mm}$ \\
circunferencia extremidad previa al muñón & $6,0 \mathrm{~mm}$ \\
distancia a la articulación más cercana & $31 \mathrm{~mm}$ \\
altura de extremidad amputada & $7 \mathrm{~mm}$ \\
peso de la extremidad amputada & $2 \mathrm{~g}$ \\
\hline
\end{tabular}




\section{RESULTADOS}

\section{Corrección de eje y rotación corporal Falco sparverius}

Posteriormente al uso de la prótesis, el ejemplar de cernícalo americano presentó una corrección total de eje corporal y de inclinación corporal (Figura 3), permitiendo al ejemplar mantenerse erguido por periodos prolongados de tiempo. La prótesis se mantuvo fija a través del socket diseñado y paulatinamente el ejemplar la fue incorporando dentro de sus actividades cotidianas, incluyendo el desplazamiento.

\section{Caso lechuza de campanario (Tyto alba)}

La lechuza de campanario es un ave rapaz nocturna con una amplia distribución en el continente, y se considera con una amplia capacidad de reproducción. Dicha especie no se encuentra en alguna categoría de riesgo en la Norma Oficial Mexicana y dentro de la

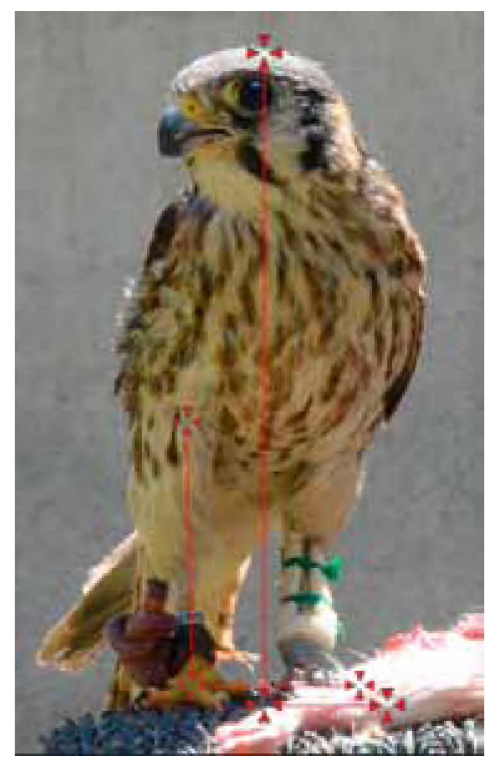

Figura 3. Ejemplar de cernícalo americano (Falco sparverius) con prótesis tarsometatarsal. Se muestran los ángulos de las inclinaciones en el eje longitudinal y la extremidad derecha.

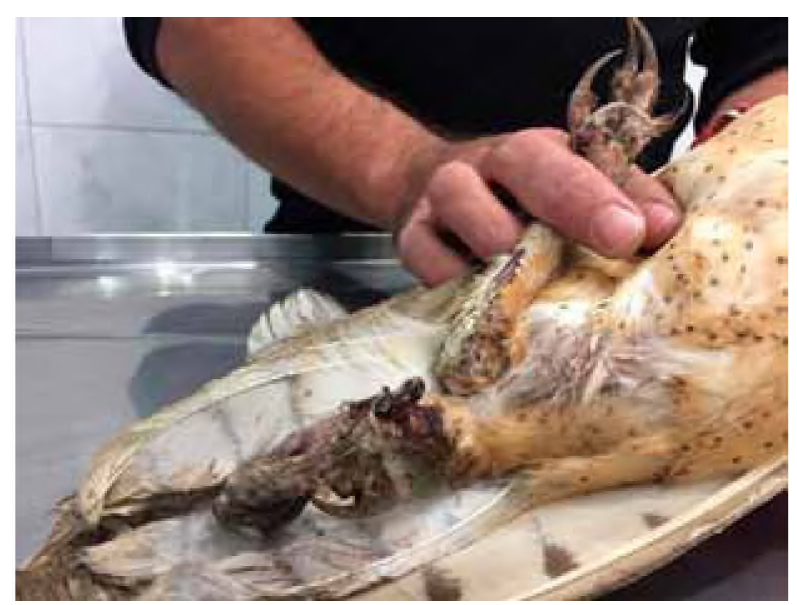

Figura 4. Fractura expuesta en la extremidad inferior izquierda de lechuza de campanario (Tyto alba).
Tabla 2. Medidas de superficie de muñón y superficie y peso perdido (Tyto alba)

\begin{tabular}{lc}
\hline circunferencia del muñón & $13 \mathrm{~mm}$ \\
circunferencia extremidad previa al muñón & $11 \mathrm{~mm}$ \\
distancia a la articulación más cercana & $35 \mathrm{~mm}$ \\
altura de extremidad amputada & $35 \mathrm{~mm}$ \\
peso de la extremidad amputada & $7 \mathrm{gr}$ \\
\hline
\end{tabular}

lista roja de especies amenazadas IUCN se encuentra en la categoría de menor preocupación, sin embargo, dentro de la lista de especies amenazadas (CITES) se encuentra en el apéndice II.

Estudios sobre el monitoreo de especies nocturnas han identificado un descenso en las poblaciones de lechuza de campanario en países bajos de Europa occidental, causada por la intervención humana (persecución, mortalidad vial y pesticidas), así como la pérdida de hábitat en zonas agrícolas y la urbanización de zonas rurales ${ }^{24}$. En Estados Unidos la lechuza de campanario se ha establecido como una especie amenazada o en alguna categoría de riesgo en aproximadamente 16 estados del país ${ }^{25}$

El ejemplar hembra de lechuza de campanario (Tyto alba) fue recibido en las instalaciones del Centro de Conservación de Vida Silvestre "Konkon", posteriormente a un rescate realizado por la Procuraduría Federal de Protección al Ambiente (Delegación Puebla), el ejemplar presentaba una fractura expuesta en el tercio distal del tarsometatarso (Figura 4). Además de la fractura, el ejemplar no presentó lesiones ni deficiencias de otro tipo.

Se realizó una evaluación de circulación sanguínea y sensibilidad en los dígitos afectados para identificar el daño general provocado por la lesión más allá de la pérdida de continuidad del tarsometatarso; se comprobó una falta de sensibilidad e irrigación sanguínea en los dígitos y se procedió a amputar la extremidad en colaboración con el Hospital Veterinario de Pequeñas Especies de la Universidad de Puebla, bajo el mismo procedimiento descrito en el primer caso.

El ejemplar fue sedado con Ketamina $10 \mathrm{mg} / \mathrm{kg}$ y Midazolam 0,25 mg/kg (aplicación intramuscular). Se mantuvo anestesiado con isoflurano con sonda endotraqueal CAM 1,44\%, $02200 \mathrm{ml} / \mathrm{kg} / \mathrm{min}$, VPP $8 \mathrm{rpm} /$ 8-10 $\mathrm{cm}$ de agua. Se mantuvo una terapia de líquidos $20 \mathrm{ml} / \mathrm{kg} / \mathrm{h}$, solución de $\mathrm{NaCl}(0,9 \%)$, las respuestas cardiaca, respiratoria y temperatura corporal fueron monitoreadas durante el procedimiento quirúrgico.

Las constantes fisiológicas se mantuvieron en rango y el ejemplar permaneció estable durante el procedimiento, la amputación se realizó 1,5 centímetros por encima de la fractura expuesta. Una vez finalizada la amputación, la incisión fue cerrada con monofilamento absorbible y después de la cirugía el ejemplar fue medicado con Meloxicam $(0,5 \mathrm{mg} / \mathrm{kg})$ y Tramadol $(5 \mathrm{mg} /$ $\mathrm{kg}$ ) en dosis orales.

El ejemplar no presentó inclinación de eje corporal en el plano sagital compensatorio a la pérdida de la 


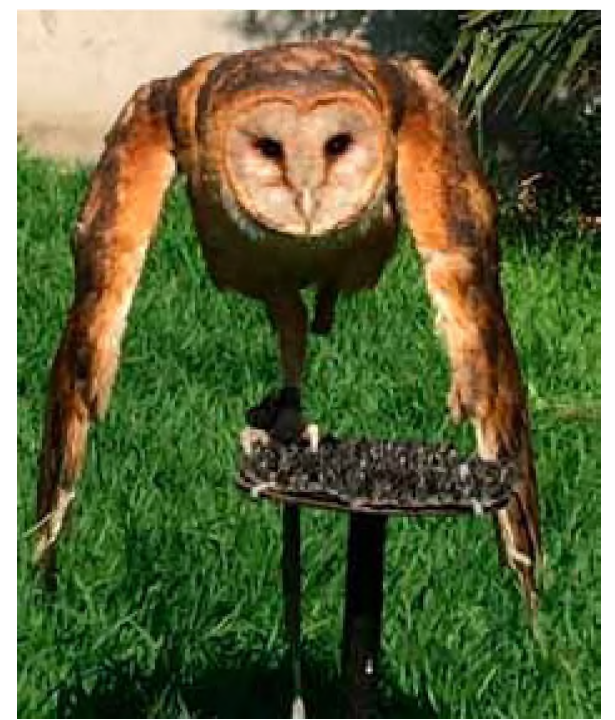

Figura 5. Ejemplar de lechuza de campanario (Tyto $a l b a$ ) con amputación tarso-metatarsal, previo a la prueba de prótesis. Se observa la inclinación del eje en el plano frontal y la extensión de las alas para mantener el equilibrio

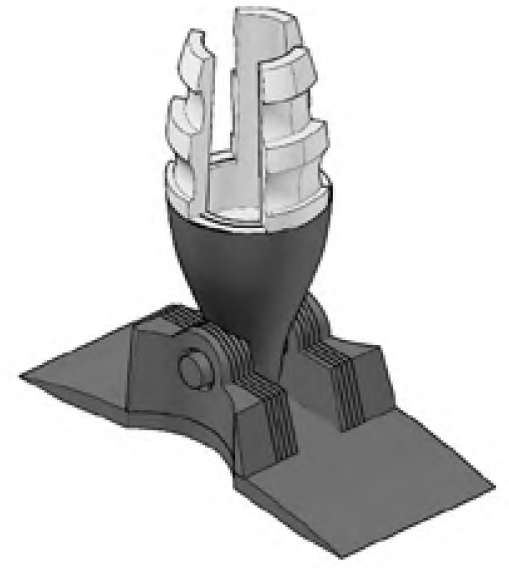

Figura 6. Modelo 3D de prótesis para lechuza de campanario, el material blanco corresponde al plástico flexible y el material gris a plástico rígido.

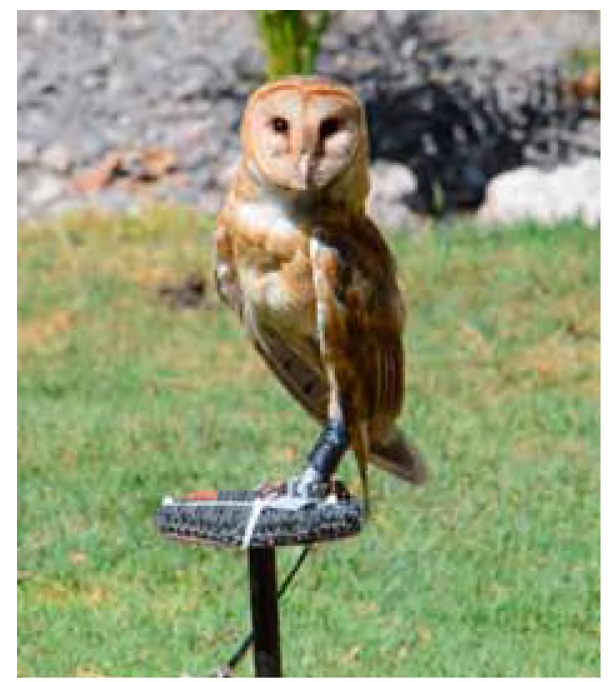

Figura 7. Lechuza de campanario en posición erguida con las alas encogidas. extremidad. Mantenía el equilibrio alterando el plano frontal, inclinando el eje hacia el frente y extendiendo las alas (Figura 5).

\section{Toma de medidas de muñón}

Se tomaron las medidas de la extremidad amputada, analizando la circunferencia del muñón, circunferencia en la extremidad anterior al muñón, distancia a la articulación más cercana, la superficie disponible para fijación de la prótesis, la altura faltante de la extremidad y el peso faltante de la extremidad, obteniendo los resultados indicados en Tabla 2. Con los datos obtenidos, se iniciaron los diseños de prótesis, así como los diseños y pruebas de socket para fijación de la prótesis.

\section{Diseño de prótesis (Tyto alba)}

Se diseñó prótesis en 3D utilizando el programa AutoCAD, desarrollando un total de 3 diseños de socket y 7 diseños de poste/base para lograr una correcta fijación (Figura 6).

\section{Impresión de prótesis}

Se utilizó una impresora 3D marca Ultimaker Modelo 3 de doble extrusor, la articulación entre el poste y la base o pie, se incluyó dentro del mismo proceso de impresión, para evitar un generar un punto débil al montar y desmontar la articulación. El peso de la prótesis se reguló disminuyendo el relleno de la impresión.

La impresión del poste y del socket se realizó con filamento de plástico rígido y filamento de plástico flexible respectivamente (Figura 6).

\section{Resultados de prueba de prótesis}

Durante el periodo de prueba, el ave se quitó constantemente la prótesis y se le volvió a colocar todas las veces. Inicialmente, el ave reposaba recostada. Posteriormente empezó a incorporar el uso de la prótesis para desplazarse, perchar y sujetar su alimento. La prótesis permitió que el ave recuperara su postura natural, recogiendo las alas al ya no necesitarlas para mantener el equilibrio (Figura 7). Gracias a la articulación, también utilizó la prótesis para impulsarse a saltar.

El plumaje del ejemplar mejoró considerablemente con una variación de alimento entre rata y ratón.

\section{Conclusiones}

Las prótesis diseñadas y desarrolladas específicamente para un ejemplar de cernícalo americano (Falco sparverius) y de lechuza de campanario (Tyto alba) demostraron ser eficientes para la corrección de inclinación corporal, así como para la redistribución del peso corporal del ejemplar, logrando reducir las lesiones de presión, como pododermatitis.

Los materiales utilizados para el desarrollo de la prótesis han funcionado de forma eficiente. Después de más de 6 meses de uso, la prótesis no ha sufrido ningún tipo de daño, a pesar de estar expuesta a las inclemencias del clima y en algunas ocasiones a gol- 
pes producidos por el mismo ejemplar en el proceso de acostumbramiento al uso de la prótesis.

La utilización de prótesis impresas en 3D significa una reducción en costos de materiales y un menor tiempo de trabajo, ya que no se deben realizar moldes para cada modificación de prótesis y pueden representar una buena alternativa para el mantenimiento en cautiverio de ejemplares amputados que no pueden ser reintegrados al medio ambiente.

La integración de una articulación en el proceso de impresión confiere una mayor resistencia a los movimientos de las aves y permite que incorporen el uso de la prótesis de forma más natural en actividades cotidianas como desplazarse, alimentarse y perchar.

Agradecimientos. A la Secretaría de Educación Pública de México, por el apoyo y financiamiento del proyecto. A los alumnos de Biología, Veterinaria y Zootecnia Nefris Xel Herrera, Argelia Mariel Mino, Aislim Gómez, Alejandra Patricia Arcos, Michelle Esther Arevalo, Paulina Plata Camarena, Daniela Cruz Hernández, Guadalupe Lima, Aline Juárez Balboa y Verónica Patricia Guevara por su apoyo. A la Arq. Begoña Calderón por su asesoría y al Arq. Jaime Adrián Alcalá por su guía altruista.

\section{REFERENCIAS}

1. Stange EA, Oidor MA, Ramírez JA. 2018. Physiotherapy in tibiotarsal fracture rehabilitation in raptors. Journal of Wildlife Rehabilitation 37: 3, 17-21.

2. Herrera CN, Oidor MA, Stange EA. 2019. Case study: rehabilitation of a red-tailed hawk (Buteo jamaicensis) with a severe neurological disorder. Journal of Wildlife Rehabilitation 39: 1, 7-10.

3. Harrison G, Lightfoot T. 2005. Clinical Avian Medicine, Spix Publishing, USA, $422-427$ p.

4. Bedrosian B, Pierre SA. 2007. Frequency of injuries in three raptor species wintering in northeastern Arkansas. Wilson Journal of Ornithology 119: 296-298.

5. Strobel B, Haralson C. 2009. Foraging by a red shouldered hawk (Buteo lineatus) with severe bill damage. Bulletin of Texas Ornithological Society 42: 96-98.

6. Blodget B, Davis W, Pokras M. 1990. Bald eagle survives two years in wild with one foot. Journal of Field Ornithology 61: 76-78.

7. Murza G, Bortolotti G, Dawson R. 2000. Handicapped American Kestrels: needy or prudent foragers? Journal of Raptor Research 34: 137-142.

8. Dwyer J. 2006. Electric shock injuries in a Harris's Hawk population. Journal of Raptor Research 40: 193-199.

9. Dirzo R. 1990. La biodiversidad como crisis ecológica actual, ¿qué sabemos?. Número Especial de la Revista Ciencias (UNAM, México) 4: 48-55.
10. Ehrlich A, Ehrlich P. 1992. Causes and consequences of the disappearance of biodiversity. Revista Ciencia www. biodiversidad.gob.mx.

11. Soulé R. 1992. Conservation Biology Today: the most pressing questions. In: Sarukhán, J. y Dirzo R.: México ante los retos de la biodiversidad, Conabio, 1992.

12. Ritchie B, Harrison G, Harrison L. 1994. Avian medicine: principles and application. Wingers Publishing ISBN 0-9636996-0-1.USA.

13. Samour J. 2016. Avian Medicine (Tercera ed.), Elsevier, USA and Biodiversidad, Conabio, México.

14. Coles B. 2007. Essentials of avian medicine and surgery, 3ra. Ed., Blackwell Publishing, USA, 54 p.

15. Galicia A, Hernandez V, Delcastillo L, Samour J. 2018 Design and use of a 3D prosthetic leg in a red-lored amazon parrot (Amazona autumnalis). Journal of Avian Medicine and Surgery 32: 2, 133-137.

16. Marcellin LD, Drum M, Levine D, McDonald S. 2015. Orthoses and exoprostheses for companion animals. Vet Clin North Am Small Anim Pract 45: 1, 167-183.

17. Burke H, Swaim S, Amalsadvala T. 2002. Review of wound management in raptors. Journal of Avian Medicine and Surgery 16: 3, 180-191.

18. Olgun ED, Özsoy S, Aydin D, Hasimbegoviç H, Dönmez K. 2012. Partially forelimb amputation and application of an artificial limb (prosthetics) in a free-ranging Red Deer (Cervus elaphus). Kafkas Üniv.Vet. Fakült Derg 18: 2, 345-350.

19. Herbert N, Simpson D, Spence W, Ion W. 2005. A preliminary investigation into the development of 3 -D printing of prosthetic sockets. Journ Rehabilit Research \& Development 42 : 2, 141-146.

20. Marshall J. 2020. The history of prosthetics. Orthotics and Prosthetics. http://unyq. com/the-history-of-prosthetics. Accessed on August 10, 2020.

21. Henny C, Brady G. 1994. Partial migration and wintering localities of American kestrels nesting in the Pacific Northwest. Soc for Northwest Vertebr Biol: 37-43.

22. Smallwood J et al. 2009. Why are American kestrel (Falco sparverius) populations declining in North America? Evidence from nest-box programs. Journal of Raptor Research 43: 4, 274-282.

23. Medica D, Clauser R, Bildstein K. 2007. Prevalence of West Nile virus antibodies in a breeding population of American kestrels (Falco sparverius) in Pennsylvania. Journal of Wildlife Diseases 43: 3, 538-541.

24. Debrujin O. 1994. Population ecology and conservation of the barn owl (Tyto alba) in farmland habitats in Liemers and Achterhoek (the Netherlands). ARDEA 82: 1-109.

25. Martin J, Raid R, Branch L. 2005. http://www.wildflorida.org/bba. Barn Owl (Tyto alba), Wec 185/UW 216. 